\title{
Earth Resources Observation and Science (EROS) Center Popular Web Sites
}

\section{USGS EROS Center}

eros.usgs.gov

EROS Image Gallery

eros.usgs.gov/imagegallery

EarthNow! Landsat Viewer

earthnow.usgs.gov

Earth Explorer

earthexplorer.usgs.gov

Global Visualization Viewer

glovis.usgs.gov

\section{The National Map}

nationalmap.gov

USGS Land Remote Sensing

remotesensing.usgs.gov

Landsat and Landsat

Data Continuity Mission

(LDCM) Programs

landsat.usgs.gov

Remote Sensing Technologies

calval.cr.usgs.gov

Commercial Remote Sensing

Space Policy (CRSSP)

crssp.usgs.gov

TerraLook Imagery

terralook.cr.usgs.gov

Land Cover Institute

landcover.usgs.gov

GISDATA Map Studio

gisdata.usgs.net
Light Detection and Ranging

(LIDAR) Information Coordination and Knowledge

lidar.cr.usgs.gov

Interferometric Synthetic

Aperture Radar (InSAR) and

Land Surface Deformation

edc2.usgs.gov/Geo_Apps/index.php

United Nations Environment Programme/Global Resource Information Database (GRID)

na.unep.net

USGS International Program

international.usgs.gov

National Wetlands

Research Center

www.nwrc.usgs.gov

USGS Science

usgs.gov

biology.usgs.gov

energy.usgs.gov

geography.usgs.gov

geology.usgs.gov

minerals.usgs.gov

water.usgs.gov

USGS Natural Hazards

usgs.gov/hazards

earthquake.usgs.gov

landslides.usgs.gov

fire-research.cr.usgs.gov

volcanoes.usgs.gov

Hazards Data Distribution

System (HDDS)

hdds.usgs.gov/EO
NASA Land Processes

Distributed Active

Archive Center (LP DAAC)

lpdaac.usgs.gov

ASK USGS

ask.usgs.gov

USGS Library

library.usgs.gov

USGS Store

store.usgs.gov/b2c_usgs/b2c/start.do

USGS Publications Warehouse

infotrek.er.usgs.gov/pubs

USGS State Offices

usgs.gov/contact_us

USGS and Science Education

education.usgs.gov

USGS Employment

usgs.gov/ohr

my.usajobs.gov

USGS LandsatLook Viewer

landsatlook.usgs.gov

For more information

please contact:

U.S. Geological Survey

Earth Resources Observation and Science (EROS) Center

47914 252nd Street

Sioux Falls, SD 57198-0001

Toll Free:

800-252-4547

Telephone:

605-594-6151

Fax: 605-594-6589

E-mail: custserv@usgs.gov 